\title{
Nichtalkoholische Steatohepatitis (NASH) und alkoholische Steatohepatitis (ASH)
}

\section{Einleitung}

Eine Fettlebererkrankung kann durch übermäßigen Alkoholkonsum (Alcoholic Liver Disease, ALD), Dysmetabolismus oder andere Umwelteinflüsse ausgelöst werden, die zu Steatohepatitis führen und sich zu chronischem Leberversagen weiterentwickeln können, insbesondere wenn spezifische genetische Determinanten vorhanden sind. Während das metabolische Syndrom und der Alkoholkonsum die beiden Hauptursachen für chronische Lebererkrankungen sind, überwiegt oft eine der beiden Erkrankungen und die andere fungiert als Cofaktor. Adipositas und Alkohol wirken synergistisch im Hinblick auf das Risiko einer Fibroseprogression, Leberkarzinogenese und Erhöhung der Mortalität. Genetische Polymorphismen können den Krankheitsverlauf stark beeinflussen.

Die nichtalkoholische Fettleberkrankung (NAFLD) beschreibt ein Kontinuum von der einfachen nichtalkoholischen Fettleber (NAFL) zur nichtalkoholischen Fettleberhepatitis oder Steatohepatitis (NASH) und $\mathrm{NASH}$-Fibrose. NAFLD hat einen variablen Verlauf, kann aber genauso wie die alkoholische Fettleber, die sogenannte ALD, zu Leberzirrhose und Leberkrebs, dem hepatozellulären Karzinom (HCC), seltener auch dem intrahepatischen cholangiozellulären Karzinom (iCCa) führen. Es sind derzeit noch keine Medikamente zur Behandlung der NAFLD oder NASH zugelassen. Allerdings befinden sich zahlreiche Substanzen in der fortgeschrittenen klinischen Prüfung. Entsprechende Einzelwirkstoffe oder Kombinationstherapien sollen den Progress der Krankheit stoppen und im besten Fall pathologische Leberanomalien rückgängig machen. Die Prävalenz der NAFLD steigt weltweit an - parallel zum Anstieg von Typ-2-Diabetes (T2D) mellitus, Adipositas und dem metabolischen Syndrom. Gewichtsreduktion, gesunde Ernährung und viel Bewegung sind entsprechend der gültigen Leitlinien die beste Prävention und Therapie.

\author{
Definition
}

Um die Diagnose der nichtalkoholischen Fettlebererkrankung NAFLD zu stellen, müssen Belege für eine Leberverfettung entweder durch eine geeignete Bildgebung (Sonografie, Magnetresonanztomografie) oder eine Leberhistologie vorliegen. Andere Ursachen einer Leberverfettung (d.h. Differenzialdiagnosen), zum Beispiel erheblicher Alkoholkonsum (d.h. durchschnittlich > $10 \mathrm{~g}$ pro Tag bei Frauen und $>20 \mathrm{~g}$ pro Tag bei Männern), langzeitige Einnahme steatogener Medikamente oder genetische Erkrankungen, müssen zudem ausgeschlossen werden [3, 6, 7].

\section{Pathophysiologie/ Epidemiologie}

NAFLD bezeichnet ein ganzes Spektrum von histologischen Leberveränderungen, die von der einfachen Steatose (NAFL) bis hin zur Steatohepatitis (Verfettung und Entzündung, NASH) mit/ohne Fibrose, zur fettleberbedingten („kryptogenen“) Zirrhose und zum HCC, seltener auch dem iCCa, reichen. Ein dysmetabolisches Milieu, allerdings ohne übermäßigen Alkoholkonsum und andere konkurrierende Ätiologien einer chronischen Lebererkrankung stellen die Grundlage der NAFLD dar [7]. NAFLD wird als hepatische Manifestation des metabolischen Syndroms angesehen, kann aber auch unabhängig davon auftreten. Die NAFLD-Prävalenz in der Allgemeinbevölkerung liegt weltweit bei ca. 20$30 \%$, in Deutschland bei ca. $25 \%$ und ist verbunden mit einer erhöhten Mortalität, bedingt durch kardiovaskuläre Erkrankungen, Tumorerkrankungen und die Lebererkrankung an sich [1,2]. Pathophysiologisch ist die NASH durch eine hepatozelluläre Schädigung, angeborene Immunzellenvermittelte Entzündung und progressive Leberfibrose gekennzeichnet. Die Mechanismen der NASH scheinen zudem mit einem pro-inflammatorischen Mikroenvironment zusammenzuhängen, das vor allem durch die sogenannte Lipotoxizität induziert wird [5].

\section{Diagnostik}

Der transabdominelle Ultraschall sollte als primäre Bildgebung bei Patienten mit V.a. NAFLD oder ALD eingesetzt werden [7]. Ein Ultraschall mit Steatosis hepatis erlaubt keinen Ausschluss einer Fettleberhepatitis und damit keine Unterscheidung zwischen ALD, nichtalkoholischer Fettleber (NAFL) und der nichtalkoholischen Fettleberhepatitis (NASH). Die ultraschallbasierte Scherwellen-Elastografie kann zum Ausschluss einer fortgeschrittenen Leberfibrose und Leberzirrhose herangezogen werden [7, 12]. Eine Unterscheidung zwischen Steatohepatitis und Fibrose ist mittels Elastografie ebenfalls nicht möglich. Die Elastografie kann deshalb für den Nachweis einer Fibrose nur mit Einschränkung verwendet werden. Hierfür ist weiterhin eine Leberbiopsie als Goldstandard erforderlich [7]. In einer kürzlich publizierten Studie konnten mittels WholeExom-Sequencing genetische Assoziationen zwischen PNPLA3 und TM6SF2 mit fortgeschrittener Fibrose an einer histologisch gesicherten NAFLD-Kohorte im Vergleich zu einer großen Populationskontrolle bestätigt werden [8]. Klinisch sinnvoll ist der Einsatz von Fibrose-Markern und -Scores wie NFS oder FIB-4 zur nichtinvasiven Identifizierung von NAFLD-Patienten mit Grad 3 und 4 Fibrose $[6,12]$.

\section{Therapie}

Das therapeutische Ziel bei ALD ist die Alkoholabstinenz. Das Ziel bei der NAFLD ist die Reduktion der Insulinresistenz mit verminderten kardiovaskulären Endorganschäden und verlängertem Überleben. Durch Lebensstiländerungen, die auf moderater Gewichtsreduktion und Steigerung der körperlichen Aktivität beruhen, kann die Progression einer Insulinresistenz verhindert oder verzögert werden. Eine regelmäßige körperliche Aktivität senkt das Risiko für NAFLD. Eine erhöhte Kalorienaufnahme ist dagegen ein Risikofaktor in der NAFLD-Pathogenese [7].

Für die Indikation ALD und NAFLD gibt es bisher keine zugelassenen Medikamente. 
Auf eine generelle Einnahme von Substanzen wie Ursodesoxycholsäure, Pioglitazon, Metformin, Silymarin oder Pentoxifyllin sowie Nahrungsergänzungsmitteln (z.B. Vitamin E oder Omega-3-Fettsäuren) sollte aufgrund der aktuellen Datenlage zur Behandlung der NAFLD und ALD verzichtet werden. Aufgrund der vorteilhaften Wirkungen auf NASH sollten nichtzirrhotische NAFLD-Patienten mit T2D Metformin plus Glucagon-like Peptid 1 (GLP-1)-Agonisten (z. B. Liraglutid oder Semaglutid) nehmen. Die Verwendung von natriumabhängigen Glucosetransporter 2 (SGLT2)-Inhibitoren, z.B. Empagliflozin und Dapagliflozin oder das Thiazolidindion Pioglitazon können bei diesen Patienten ebenfalls in Betracht gezogen werden.

Patienten mit NASH-assoziierter Leberzirrhose und T2D mit kompensierter Leberzirrhose (Stadium Child A) und normaler Nierenfunktion dürfen Metformin erhalten. Zur möglichen Anwendung von GLP1-Agonisten, SGLT2-Hemmern oder Pioglitazon bei NASH-assoziierter Leberzirrhose liegen derzeit keine ausreichenden Erfahrungen vor.

Andere Antidiabetika wie Metformin, Dipeptidyl-Peptidase-IV-Hemmer oder Insulin haben bisher keine spezifischen Vorteile hinsichtlich der NAFLD-Therapie gezeigt. Große retrospektive Studien lassen vermuten, dass der Metformin-Einsatz bei NAFLD-Patienten ein verringertes HCC-Risiko zeigt [10]. Auch bei Patienten mit $\mathrm{NASH}$-assoziierter kompensierter Zirrhose (Child A) ist Metformin zur Behandlung von Diabetes mit einem verringerten Risiko für Leberdekompensation und HCC verbunden (bei normaler Nierenfunktion kann eine Dosis von $2 \mathrm{~g}$ pro Tag verwendet werden). Metformin ist kontraindiziert bei einer GFR unter $30 \mathrm{ml} / \mathrm{min}$. Allerdings gibt es bisher keine prospektiven kontrollierten Studien zum Einsatz von Metformin bei Leberzirrhose.

Da Fettstoffwechselstörungen wie die familiäre Hypercholesterinämie, Hypertriglyzeridämie, Lipoprotein(a)-Erhöhung oder isolierte HDL-Cholesterinsenkung ein stark erhöhtes Risiko für Herz-Kreislauf-Erkrankungen darstellen und NAFLD das Risiko für Herz-Kreislauf-Erkrankungen unabhän- gig von einer Fettstoffwechselstörung erhöhen könnte, sollte eine Stoffwechselstörung wirksam behandelt werden [11].

Aufgrund der günstigen Wirkung auf $\mathrm{NASH}$ werden bei nichtzirrhotischen NAFLD-Patienten mit Adipositas und Indikation zur medikamentösen Gewichtsreduktion Glucagon-like Peptid 1 (GLP-1) Agonisten, z. B. Liraglutid, verwendet. Das für die Behandlung der Adipositas zugelassene Orlistat kann bei übergewichtigen und adipösen Patienten mit NASH in nahezu gleicher Weise eingesetzt werden. Bei Patienten mit Adipositas > Grad II (BMI $>35 \mathrm{~kg} / \mathrm{m}^{2}$ ) sollte eine bariatrische Operation erfolgen, wenn die konservativen Maßnahmen fehlschlagen. Der Konsum von Kaffee kann aufgrund seiner hepatound kardioprotektiven Effekte empfohlen werden. NAFLD-Patienten sollten auf Alkohol verzichten und nicht rauchen $[2,7]$.

Innovative Therapien zielen auf Stoffwechselwege, Insulinresistenz, Apotose, Rekrutierung oder Aktivierung von Entzündungszellen, die Darm-Leber-Achse, Matrixexpression oder Matrixmetabolismus ab. Während einige Wirkstoffkandidaten in klinischen Studien der Phase 2 oder 3 versagten (z. B. Selonsertib oder Elafibranor), stützen vielversprechende Ergebnisse mit dem Farnesoid-X-Rezeptor-Agonisten Obeticholsäure, dem Pan-PPAR-Agonisten Lanifibranor und dem ChemokinrezeptorCCR2/CCR5-Inhibitor Cenicriviroc die Erwartung einer wirksamen pharmakologischen Therapie der Leberfibrose. Die größten Aussichten bietet die Bekämpfung der NAFLD-assoziierten Fibrose auf unterschiedlichen Wegen durch eine Kombination der medikamentösen Ansätze mit Änderungen des Lebensstils.

Therapeutische Interventionen zur Bekämpfung von ALD sind dagegen sehr begrenzt, wie z.B. der Einsatz von Kortikosteroiden. Innovative Mechanismen zur Prävention von ALD durch essbare Nahrungspflanzen umfassen folgende hepatoprotektive Mechanismen: Antioxidation, Entzündungshemmung, Lipidregulierung, Hemmung der Apoptose, Modulation der Zusammensetzung der Darmmikrobiota und antifibrotische Ansätze.

\section{Ausblick}

Die Humangenetik bietet einen neuen Ansatz zur Entwicklung von Therapeutika für $\mathrm{ASH}$ und $\mathrm{NASH}$, der möglicherweise $\mathrm{Er}$ gebnisse vorhergesagt und Stärken sowie Schwächen von innovativen medikamentösen Therapien in klinischen Studien offenlegt. Darüber hinaus detektiert die Molekulargenetik neue Wirkstofftargets und hat bereits zur Entwicklung der ersten präzisionsmedizinischen Ansätze für chronische Lebererkrankungen geführt. Es gilt, die genetischen Risikovarianten zu identifizieren und Steatohepatitis zu heilen, indem ätiologische Faktoren minimiert werden. Angesichts der gemeinsamen genetischen Pathophysiologie von ASH und NASH besteht auch die Hoffnung, dass einige der für NASH entwickelten neuartigen Ansätze zur Behandlung von ALD und ASH wiederverwendet werden können. Hier sind zusätzliche Studien erforderlich.

\section{Fazit}

NAFLD ist mittlerweile die häufigste Lebererkrankung in Deutschland. Die Prävention von Übergewicht und Bewegungsmangel zielt auf die wichtigsten Risikofaktoren ab. Körperliche Aktivität führt nachweislich auch ohne absolute Reduktion des Körpergewichts zu einer Besserung der Fettleber. Aktuell gibt es keine für die NASH oder NAFLD sowie ALD zugelassene spezifische medikamentöse Therapie.

\section{Interessenkonflikt}

Die Autorin erhielt Zuwendungen von folgenden Firmen für Vorträge und/oder Beratung: Abbvie, Bristol Myers Squibb, Falk Foundation, Gilead, Intercept, Merz, Norgine, Pfizer. In Bezug auf diesen Text bestehen aus Sicht der Autorin keine signifikanten Interessenkonflikte.

Autorin

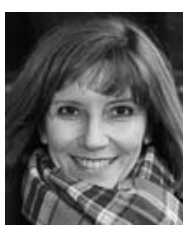

Elke Roeb

Univ.-Prof. Dr. med, MHAC, Gastroenterologie, Medizinische Klinik II, Universitätsklinikum UKGM, Justus-LiebigUniversität Gießen 


\section{Korrespondenzadresse}

Univ.-Prof. Dr. med. Elke Roeb, MHAC

Gastroenterologie, Medizinische Klinik II

Universitätsklinikum UKGM

Justus-Liebig-Universität Gießen

Klinikstr. 33

35392 Gießen

Deutschland

elke.roeb@innere.med.uni-giessen.de

\section{Literatur}

[1] Byrne CD, Targher G. NAFLD: a multisystem disease. J Hepatol 2015; 62: S47-S64

[2] Byrne CD, Targher G. EASL-EASD-EASO Clinical Practice Guidelines for the management of non-alcoholic fatty liver disease: is universal screening appropriate? Diabetologia 2016; 59: 1141-1144

[3] European Association for the Study of the Liver (EASL); European Association for the Study of Diabetes (EASD); European Associa- tion for the Study of Obesity (EASO). EASLEASD-EASO Clinical Practice Guidelines for the management of non-alcoholic fatty liver disease. J Hepatol 2016; 64: 1388-1402

[4] Friedman SL, Neuschwander-Tetri BA, Rinella $M$, Sanyal AJ. Mechanisms of NAFLD development and therapeutic strategies. Nat Med 2018; 24: 908-922

[5] Ibrahim SH, Hirsova P, Gores G]. Non-alcoholic steatohepatitis pathogenesis: sublethal hepatocyte injury as a driver of liver inflammation. Gut 2018; 67: 963-972

[6] Roeb E. Fettleber. In: Lammert F, Lynen Jansen P, Lerch M (Hrsg.). Weissbuch Gastroenterologie 2020/2021. Erkrankungen des Magen-Darm-Traktes, der Leber und der Bauchspeicheldrüse - Gegenwart und Zukunft. Berlin, Boston: De Gruyter; 2019: 7681

[7] Roeb E, Steffen HM, Bantel $\mathrm{H}$ et al. [S2 k Guideline non-alcoholic fatty liver disease]. Z Gastroenterol 2015; 53: 668-723
[8] Sanyal A], Chalasani N, Kowdley KV et al.; NASH CRN. Pioglitazone, vitamin E, or placebo for nonalcoholic steatohepatitis. N Engl J Med 2010; 362: 1675-1685

[9] Sung KC, Wild SH, Byrne CD. Resolution of fatty liver and risk of incident diabetes. J Clin Endocrinol Metab 2013; 98: 3637-3643

[10] Fujiwara N, Friedman SL, Goossens N, Hoshida Y. Risk factors and prevention of hepatocellular carcinoma in the era of precision medicine. J Hepatol 2018; 68: 526-549

[11] Roeb E, Geier A. Nonalcoholic steatohepatitis (NASH) - current treatment recommendations and future developments. Z Gastroenterol 2019; 57: 508-517

[12] European Association for the Study of the Liver; List of panel members (alphabetical order), Berzigotti A, Boursier J, Castera L, Cazzagon N, Friedrich-Rust M, Petta S, Thiele M, Tsochatzis E. Easl Clinical Practice Guidelines (Cpgs) On Non-Invasive Tests For Evaluation Of Liver Disease Severity And Prognosis 2020 Update. J Hepatol 2021; 75: 659-689 